\title{
Probiotic Bifidobacterium breve Prevents Memory Impairment Through the Reduction of Both Amyloid- $\beta$ Production and Microglia Activation in APP Knock-In Mouse $^{1}$
}

\author{
Mona Abdelhamid ${ }^{\mathrm{a}}$, Chunyu Zhou ${ }^{\mathrm{a}}$, Kazuya Ohno ${ }^{\mathrm{b}}$, Tetsuya Kuhara ${ }^{\mathrm{b}}$, Ferdous Taslima ${ }^{\mathrm{a}}$, \\ Mohammad Abdullah $^{\mathrm{a}, \mathrm{c}}$, Cha-Gyun Jung ${ }^{\mathrm{a}, *}$ and Makoto Michikawa ${ }^{\mathrm{a}, *}$ \\ ${ }^{a}$ Department of Biochemistry, Graduate School of Medical Sciences, Nagoya City University, Nagoya, Japan \\ ${ }^{\mathrm{b}}$ Next Generation Science Institute, Morinaga Milk Industry Co., Ltd., Zama, Japan \\ ${ }^{\mathrm{c}}$ Department of Neuroscience, Mayo Clinic, Jacksonville, FL, USA
}

Accepted 19 November 2021

Pre-press 21 December 2021

\begin{abstract}
.
Background: Probiotic supplementation reestablishes microbiome diversity and improves brain function in Alzheimer's disease (AD); their molecular mechanisms, however, have not yet been fully illustrated.

Objective: We investigated the effects of orally supplemented Bifidobacterium breve MCC1274 on cognitive function and AD-like pathologies in $A p p^{N L-G-F}$ mice.

Methods: Three-month-old $A p p^{N L-G-F}$ mice were orally supplemented with $B$. breve MCC1274 for four months. The shortterm memory function was evaluated using a novel object recognition test. Amyloid plaques, amyloid- $\beta$ (A $\beta$ ) levels, $A \beta$ fibril, amyloid- $\beta$ protein precursor and its processing enzymes, its metabolic products, glial activity, and cell proliferation in the subgranular zone of the dentate gyrus were evaluated by immunohistochemistry, A $\beta$ ELISA, western blotting, and immunofluorescence staining. The mRNA expression levels of pro- and anti-inflammatory cytokines were determined by qRT-PCR analysis.

Results: We found that the oral B. breve MCC1 274 supplementation prevented memory impairment in $A p p^{N L-G-F}$ mice and decreased hippocampal $A \beta$ levels through the enhancement of the a-disintegrin and metalloproteinase 10 (ADAM10) level. Moreover, administration of the probiotic activated the ERK/HIF- $1 \alpha$ signaling pathway responsible for increasing the ADAM10 level and also attenuated microglial activation, which in turn led to reduction in the mRNA expression levels of pro-inflammatory cytokines in the brain. In addition, B. breve MCC1274 supplementation increased the level of synaptic proteins in the hippocampus.

Conclusion: Our findings support the possibility that oral $B$. breve MCC1274 supplementation might be used as a potential preventive therapy for $\mathrm{AD}$ progression.
\end{abstract}

Keywords: ADAM10, Alzheimer's disease, amyloid- $\beta$ production, Bifidobacterium breve MCC1274, ERK, glial activation, HIF-1 $\alpha$, novel object recognition, synapses

\footnotetext{
${ }^{1}$ This article received a correction notice (Erratum) with the reference: 10.3233/JAD-229022, available at http://doi.org/ 10.3233/JAD-229022.

*Correspondence to: Makoto Michikawa, MD, PhD, Department of Biochemistry, Graduate School of Medical Sciences, Nagoya City University, 1 Kawasumi, Mizuho-cho, Mizuho-ku,
}

Nagoya 467-8601 Aichi, Japan. Tel.: +81 52853 8139; Fax: +81 52841 3480; E-mail: michi@med.nagoya-cu.ac.jp and Cha-Gyun Jung, PhD, Department of Biochemistry, Graduate School of Medical Sciences, Nagoya City University, 1 Kawasumi, Mizuho-cho, Mizuho-ku, Nagoya 467-8601 Aichi, Japan. Tel.: +81 528538141 ; Fax: +81 52841 3480; E-mail: jung@med.nagoya-cu.ac.jp. 


\section{INTRODUCTION}

Alzheimer's disease (AD) is a neurodegenerative disease, accounting for $50 \%$ of all dementia cases in the world [1]. Senile plaques, formed from amyloid$\beta$ (A $\beta$ ) deposition, and neurofibrillary tangles, due to the accumulation of the abnormally tau hyperphosphorylation, are the pathological hallmarks of AD. $\mathrm{A} \beta$ is produced following cleavage of the amyloid$\beta$ protein precursor (A $\beta P P)$ by $\beta$ - and $\gamma$-secretases [2]. Alternatively, it can undergo non-amyloidogenic processing through the $\alpha$-site A $\beta P P$-cleaving enzyme $(\alpha$-secretase), including a-disintegrin and metalloproteinase 10 (ADAM10), in the middle of the $A \beta$ sequence, thereby precluding $A \beta$ production [3]. Therefore, the inhibition of $A \beta$ production and deposition is still considered one of the promising AD therapeutic approaches. Other pathogeneses of AD involve neuroinflammation, deficits in energy metabolism, and vascular degeneration [4-7]. However, current therapeutic strategies based on existing theories have not yet yielded any benefits; thus, there is an urgent need for new treatment strategies for AD.

Recently, the microbiome-gut-brain axis has been the focus of clinical research studies. The connection between the gut and the brain is achieved by one of these three pathways: direct link from the gastrointestinal tract to the intrinsic nervous system, inflammatory cytokines released by microorganisms, which modulate systemic immunity, and hormonal signaling by the hypothalamic-pituitary-adrenal axis [8-10]. Probiotics are living microorganisms that have health benefits and contribute to the immune and digestive systems [11]. Reduction of microbiota diversity can cause cerebrovascular degeneration, inflammation, tau pathology, and $A \beta$ accumulation [12-14]. Moreover, the low abundance of anti-inflammatory bacteria (Bifidobacterium) and the rising of proinflammatory bacteria (Proteobacteria) have been associated with $\mathrm{AD}$ [15]. Gut microbiota secretes some bacterial neurotransmitters; for example, Bifidobacterium species secrete gamma-aminobutyric acid (GABA) [16], which could regulate brain functions. The gut microbiome also produces some beneficial metabolites such as propionic, valeric, and butyric acids, which can inhibit inflammation and $A \beta$ accumulation [17]. It has been reported that probiotics supplementation improves brain performance and alters the gut microbiome environment, suggesting that probiotics supplementation may be a potential strategy to delay $\mathrm{AD}$ progression.
It has been reported that microbiota impairment inhibits microglial activation and its phagocytic activity of $A \beta$ [18]. Neuroinflammation mediated by microglia plays a critical role in $\mathrm{AD}$ pathogenesis. Microglia have dual functions, neuroprotective or neurotoxic effects. Activation of microglia under physiological conditions enhances $A \beta$ phagocytosis and clearance leading to reduced $A \beta$ accumulation. On the other hand, chronically activated microglia cause neuronal loss owing to their involvement in the release of proinflammatory cytokines such as IL$1 \beta$ and IL-6 [19, 20], which have been shown to be increased in the brains of $\mathrm{AD}$ patients and $\mathrm{AD}$ mouse models [21, 22].

Our previous study demonstrated that oral Bifidobacterium breve strain $\mathrm{A} 1$ (B. breve MCC1274; synonym, $B$. breve A1) supplementation rescued cognitive deficits in ddY mice induced by intracerebroventricular injection with $A \beta$, through the suppression of hippocampal inflammation and immunoreactive genes [15]. Furthermore, our recent study demonstrated that oral B. breve MCC1274 supplementation for 16 weeks enhanced the cognitive functions of subjects with mild cognitive impairment (MCI) [23]. These findings suggest that oral $B$. breve MCC1274 supplementation can be a potentially effective treatment for AD. However, the molecular mechanism(s) by which this probiotic improves cognitive function remains unknown. Since ddY mice do not show AD phenotypic features, such as $A \beta$ deposition, we could not investigate whether the oral B. breve MCC1274 supplementation is associated with $\mathrm{A} \beta$ production and deposition and glial activation in the brain. Therefore, to understand the mechanisms of action and explore the possibility of this probiotic pharmaceutical potential, this study was performed using $A p p^{N L-G-F}$ as a mouse that shows the phenotypic features of $\mathrm{AD}$ [24]. In this study, we explored the effects of the oral B. breve MCC1274 supplementation on cognitive function and pathogenesis of $A D$, including its effects on $A \beta$ production, synaptic protein levels, and glial activation, and to elucidate the mechanism by which probiotic supplementation improves cognitive function. As predicted from our previous studies, oral supplementation of this probiotic attenuated cognitive impairment in $A p p^{N L-G-F}$ mice. Furthermore, we found for the first time that oral $B$. breve MCC1274 supplementation reduced $A \beta$ production by increasing the ADAM10 level in the hippocampus, and also attenuated microgliosis accompanied by the downregulation in pro-inflammatory cytokines in 


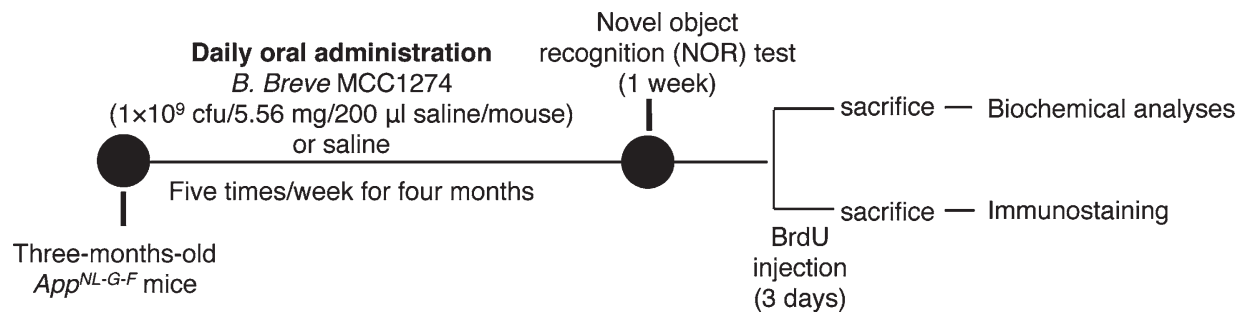

Fig. 1. Experimental procedure. Three-month-old $A p p^{N L-G-F}$ mice were assigned randomly into the vehicle and probiotic groups: the vehicle group $(n=26)$ received saline and the probiotic group $(n=26)$ was supplemented with $B$. breve MCC $1274\left(1 \times 10^{9} \mathrm{cfu} / 5.56 \mathrm{mg} / 200 \mu \mathrm{l}\right.$ saline/mouse) via oral gavage five times/week for four months. At the end of supplementation, feces were collected, and then the mice were behaviorally evaluated by novel object recognition (NOR) test or injected with BrdU. The mice were sacrificed and brains collected for biochemical analyses and immunostaining.

the brain. This study as well as our previous studies' findings suggest that oral B. breve MCC1274 supplementation might be used as a potential preventive therapy for $\mathrm{AD}$ progression.

\section{MATERIALS AND METHODS}

\section{Preparation of probiotic}

B. breve MCC1274 (stocked in Morinaga Culture Collection, Zama, Japan; synonym, B. breve A1) was provided by Morinaga Milk Industry Co., Ltd. (Zama, Japan) and prepared as described in our previous study [15]. Briefly, B. breve MCC1274 was isolated from infant feces. It was then collected by centrifugation after growing on a medium rich in yeast and glucose, then lyophilized and stored at $4{ }^{\circ} \mathrm{C}$ until use. Before oral administration to experimental mice, B. breve MCC 1274 cells were suspended in saline at a concentration of $1 \times 10^{9} \mathrm{cfu} / 200 \mu \mathrm{l} /$ day/mouse.

\section{Animal and probiotic treatments}

App knock-in (KI) mice $\left(A p p^{N L-G-F}\right)$, which overproduce $A \beta$ without overexpressing $A \beta P P$, with the Swedish, Iberian, and Arctic mutations, were obtained from RIKEN Center for Brain Science (Wako, Japan). These mice show A $\beta$ deposition starting at the age of two months until saturation at the age of seven months and exhibit memory impairment beginning at six months [24]. Three-month-old $A p p^{N L-G-F}$ mice were assigned randomly into vehicle and probiotic groups: the vehicle group $(n=26)$ received saline and the probiotic group $(n=26)$ was supplemented with $B$. breve MCC1274 $\left(1 \times 10^{9}\right.$ $\mathrm{cfu} / 5.56 \mathrm{mg} / 200 \mu \mathrm{l}$ saline/mouse) via oral gavage five times/week for four months. The mice were housed on 12-h light and dark cycle provided with water ad libitum and oriental basal diet (Oriental Yeast Co., Tokyo, Japan). All the experiments were carried out in conformity with the National Institute of Health Guide for the Care and Use of Laboratory Animals and were approved by Nagoya City University Institutional Care and Use of Laboratory Animals committee. The experimental design is shown in Fig. 1.

\section{Novel object recognition test}

After the probiotic supplementation for four months, the memory of the mice was tested using the novel object recognition (NOR) test. The NOR test is a learning and memory test to evaluate visual recognition, which is based on the principle that animals tend to explore new objects. The NOR test was carried out as reported previously [25, 26]. Briefly, it consists of three stages: a habituation stage, a training stage, and a testing stage. In the habituation stage, the mouse was allowed to explore a box without any objects for $3 \mathrm{~min}$ for three days. During the training stage, the mouse explored the box for $5 \mathrm{~min}$, which contained two familiar objects. The testing stage was $24 \mathrm{~h}$ after the training stage. In this stage, the box contained a new object (novel) and a familiar object, and the mouse was let free to explore both objects for 5 min. A video camera was used to record the exploration time in both training and testing stages. During the experiments, the objects were matched in terms of their emotional neutrality and physical complexity of the mice. A discrimination index (DI) reflects the duration of exploration for the novel object compared to the familiar object as a proportion of the total exploration time of the mouse for both objects. Exploration is defined as touching or sniffing the object. NOR analysis was performed blinded. 


\section{A $\beta$ ELISA}

Brain $A \beta$ levels were determined as described in a previous report [27]. Hippocampal and cortical tissues were homogenized and centrifuged at 100,000 rpm for $20 \mathrm{~min}$. The supernatants were used for soluble $\mathrm{A} \beta \mathrm{PP}(\mathrm{sA} \beta \mathrm{PP})$ and soluble $\mathrm{A} \beta$ measurements. The pellets were dissolved in 10 volumes of $6 \mathrm{M}$ guanidine hydrochloride, sonicated, and incubated for $1 \mathrm{~h}$ at room temperature, then centrifuged at $100,000 \mathrm{rpm}$ for $20 \mathrm{~min}$, which were used for insoluble $A \beta$ determination. $A \beta_{40}$ and $A \beta_{42}$ were determined using ELISA kits (294-64701, 290-62601 respectively, Wako Pure Chemical Industries, Osaka, Japan). The levels of $A \beta$ were normalized to the weight of the brains.

\section{Western blotting}

Hippocampal and cortical tissues were homogenized in lysis buffer containing a protease inhibitor mixture. Equal protein amounts were subjected to SDS-PAGE and immunoblotting on a membrane (IPVH00010, Millipore). This was followed by blocking and incubation with primary antibodies: anti-ADAM10 (1:1000, MAB19026, Millipore), anti-APP (1:1000, MAB348, Millipore), anti-PS1 (1:1000, MAB5232, Millipore), antiBACE1 (1:1000, MAB931, R\&D), anti- sA $\beta P P \beta$ ( $1: 1000$, \#10321, IBL), anti-6E10 (1:1000, SIG39300, Vovanc), anti-PSD95 (1:1000, \#9900, Cell signaling), anti-SYT $(1: 1000, \# 612714$, BD Transduction), anti-HIF-1 $\alpha(1: 1000, \# 3716$, Cell signaling), anti-PKC ( $1: 1000,610108$, BD Transduction), anti-phospho-PKC $(1: 1000$, \#06-822, Upstate Cell Signaling), anti-ERK $(1: 1000$, \#9102, Cell Signaling), anti-phospho-ERK $(1: 1000$, \#9101, Cell Signaling), anti-GFAP ( $1: 1000$, G3893, Sigma), anti-total tau (1:1000, 806401, Biolegend), antiphospho-tau (AT180, $1: 1000$, MN1040, Invitrogen), anti-phospho-tau (PHF1, 1:1000, MN1020, ThermoFisher scientific), anti-Iba1 (1:1000, 019-19741, Wako), and anti-actin (Proteintech Group). Washing and incubation with a proper HRP-conjugated secondary antibody was the done. Immuno Star Zeta or Immuno Star LD (295-72404, 29069904 respectively, Wako) was used for visualizing immunoreactive bands, which were imaged using an Amersham Imager 680 (GE Healthcare Life Science). The intensity of the signal was quantified using ImageJ (NIH, Bethesda, Maryland, USA).

\section{A $\beta$ immunohistochemistry}

Paraffin-fixed sagittal brain sections were processed for $A \beta$ immunohistochemistry. Sections were boiled in citrate buffer $(\mathrm{pH} 6.0,10 \mathrm{mM}$ trisodium citrate) for $5 \mathrm{~min}$ followed by cooling. After blocking with $5 \%$ goat serum (S-1000, Vector Laboratories Inc) for $30 \mathrm{~min}$, brain sections were incubated with an anti-82E1 (1:100, 10323, IBL) antibody overnight at $4{ }^{\circ} \mathrm{C}$ followed by incubation with goat anti-mouse Alexa Fluor 488 (A11029, Thermo Fisher Scientific) for $1 \mathrm{~h}$ at room temperature. Nuclei were stained with DAPI (P36931, Vector Laboratories Inc). Images were obtained using a microscope (Carl Zeiss). A $\beta$ plaques were evaluated as the percentage of the immunostained area (positive pixels) divided by the total area examined (total pixels) using ImageJ software.

\section{Immunofluorescence staining and cell proliferation analysis}

Immunofluorescence and bromodeoxyuridine (BrdU) staining were conducted using another set of mice. After the probiotic supplementation for four months, the mice ( $n=6$ per each group) were injected with $50 \mathrm{mg}$ of BrdU (027-15561. Wako) per $\mathrm{kg}$ body weight for 3 days (twice per day). The mice were anesthetized with sevoflurane, then perfused transcardially with cold PBS and 4\% PFA. The brains were quickly removed, postfixed in $4 \%$ PFA for $24 \mathrm{~h}$, and then cryoprotected for 2-3 days with $30 \%$ sucrose $\left(0.038 \mathrm{M} \mathrm{NaH}_{2} \mathrm{PO}_{4}\right.$ and 0.162 $\mathrm{Na}_{2} \mathrm{HPO}_{4}$ ). Serial coronal sections were cut on a vibratome (40 $\mu \mathrm{m}$ thickness, Leica Microsystems) and collected in a series 12 and stored in a cryoprotection solution $(30 \%$ sucrose, $150 \mathrm{mM} \mathrm{NaCl}$, and $250 \mathrm{mM}$ polyvinylpyrrolidone in $0.1 \mathrm{M} \mathrm{PB}$ ) at $-20^{\circ} \mathrm{C}$ until immunofluorescence staining for BrdU, Iba1, and GFAP. For BrdU immunofluorescence staining, the sections were boiled in a citrated buffer on slides in a pressure cooker for $5 \mathrm{~min}$, then washed in PBS. BrdU was stained using a 5-bromo-2-deoxy-uridine labeling and detection kit (11296736001, Roche) in accordance with the manufacturer's instructions. For the Iba1 and GFAP staining, sections were incubated overnight at $4{ }^{\circ} \mathrm{C}$ with the appropriate primary antibodies, namely, mouse monoclonal anti-82E1 antibody with rabbit polyclonal anti-Ibal $(1: 100$, 019-19741, Wako) or rabbit polyclonal anti-GFAP $(1: 100$, RO1003, Shima Lab) antibodies. The sections were washed and incubated with the secondary 
antibodies for $1 \mathrm{~h}$, namely, goat anti-mouse Alexa Fluor 488 or 568 and goat anti-rabbit Alexa Fluor 568 antibodies (A11029, A11031, A11036, respectively, ThermoFisher Scientific). Nuclei were stained with DAPI (P36931, Vector Laboratories Inc). Finally, images were obtained using a confocal fluorescence microscope SpinSR10 (Olympus). To quantify the BrdU-positive cells, the number of positive cells in nine sections per each animal was measured. The quantification of Iba1-positive and GFAP-positive cells was performed using Image J software in $1 \times 1$ $\mathrm{mm}^{2}$ square per field in 4 and 2 randomly selected fields in the cortex and hippocampus of each animal, respectively, and the mean was used for statistical analysis.

\section{Staining of $A \beta$ fibril}

We used Thioflavin T (ThT, \# SHBN0977, Sigma) for staining $A \beta$ fibril. Brain sections were placed in $0.5 \%$ acid alcohol for $5 \mathrm{~s}$, then rinsing in four changes of distilled water. Then the section was stained with $1 \%$ ThT for 5 min. Followed by washing well in running water and placing in $1 \%$ acetic acid for 15 min. Finally, images were obtained using a confocal fluorescence microscope SpinSR10 (Olympus). To quantify A $\beta$ fibrils; the ThT-positive plaque burdens area was measured using ImageJ software in 4 and 2 randomly selected fields in the cortex and hippocampus, respectively, per each animal. $A \beta$ fibril was evaluated as the percentage of the immunostained area divided by the total area.

\section{Quantitative real-time PCR ( $q R T-P C R)$ analysis}

Trizol (Invitrogen) was used to isolate total RNA from the hippocampal and cortical tissues according to the manufacturer's instructions. The ReverTra Ace qPCR RT Kit (FSQ-101, TOYOBO) was used for cDNA synthesis. qRT-PCR was performed with GeneAce SYBR qPCR Mix (319-07683, Nippon Gene) using the 7500 Fast Real-Time PCR System (Applied Biosystems). All samples were normalized to the corresponding internal gene control, GAPDH gene, following the manufacturer's instructions. Amplification was performed using these primers: IL-6: sense, 5'-TGATGGATGCTACCAAACTGAT3', and antisense, 5'-CTGTGACTCCAGCTTATCT CTTGGT-3'; IL-1 $\beta$ : sense, 5'-GAAGCACCAGCA CATTGCTT.

T-3', and antisense, 5'-GGAGCCTC-ATGGCCC AATTT-3'; TGF- $\beta 1$ sense, 5 '-ACTGGAGTTG-TA
CGGC-3', and antisense, 5'-GGGGCTGATCCCGT TG-3'; ADAM10 sense, 5'-CACCAAAAACACCA GCGTGC-3' and antisense, 5'-AGTGTCCCTCTT CATTCGTAGG-3'; GAPDH: sense, 5'-GCATCTT CTTGTGCAGTGCC-3', and antisense, 5'-GAGAA GGCAGCCCTGGTAAC-3'.

\section{Microbiota analysis}

All feces were collected at the end of the probiotic supplementation and used for microbiota analysis (probiotic group, $n=19$ and saline group, $n=17$ ). DNA was extracted from $20 \mathrm{mg}$ of feces using GENE PREP STAR PI-480 (Kurabo Industries Ltd., Osaka, Japan) and FastPrep-24 5G Homogenizer (MP Biomedicals, CA, USA). 16S rRNA gene sequencing was performed as previously described [28]. The sequence data obtained were analyzed using QIIME 2 version 2017.10 [29, 30]. Potentially chimeric sequences were removed with DADA2 [31], followed by trimming 30 and 90 bases of the 3' region of the forward and reverse reads, respectively. The taxonomical classification was conducted using the Naive Bayes classifier trained on the Greengenes 13.8 with a $99 \%$ threshold of an operational taxonomic unit (OTU) of 16S rRNA full-length sequences. Principal coordinate analysis (PCoA) was carried out on the basis of the weighted UniFrac distance estimated using QIIME 2 software.

\section{Statistical analysis}

Statistical analysis was performed using GraphPad Prism software (GraphPad Software, San Diego, CA). Data are presented as the mean \pm SD. Student's $t$-test was used for analyzing statistical significance. A $p$-value $<0.05$ was considered significant.

\section{RESULTS}

\section{B. breve MCC1274 supplementation prevents memory impairment in App ${ }^{N L-G-F}$ mice}

We firstly performed a NOR test, which has been previously used to characterize memory impairment in $A p p^{N L-G-F}$ mice [32,33], to determine the effects of oral $B$. breve MCC1274 supplementation on recognition ability and short-term memory in $A p p^{N L-G-F}$ mice that exhibit memory and learning deficits at seven months of age [24,34]. The probiotic and vehicle groups showed similar exploratory preferences between the two familiar objects during the train- 
ing session (Supplementary Figure 1A), suggesting that both the probiotic and vehicle groups have an equal level of interest toward the familiar objects. However, in the retention session, the vehicle group had no significant difference in exploration time for either the familiar or novel object, whereas the probiotic group had a significantly increased exploration time for the novel object compared with the familiar object (Supplementary Figure 1B). Moreover, the discrimination index (DI) was higher in the probiotics group compared to the vehicle group (Supplementary Figure 1C). This result is consistent with our previous finding that $B$. breve MCC1274 supplementation prevented memory impairment in $A \beta$-injected mice [15].

\section{B. breve MCC1274 supplementation reduces $A \beta$ production and deposition in the hippocampus}

$\mathrm{A} \beta$ deposition in the brain is a hallmark of $\mathrm{AD}$ development. Therefore, we examined $A \beta$ levels in the brains of mice to evaluate whether the preventing of memory impairment has resulted from the decrease in $A \beta$ levels. We performed immunohistochemical staining for $A \beta$ to evaluate $A \beta$ deposition in the brains. Paraffin-fixed sagittal brain sections were stained with the anti-A $\beta$ antibody (82E1) that recognizes both $A \beta_{40}$ and $A \beta_{42}$. The result demonstrated a significant reduction in the level of $A \beta$ deposition in the hippocampus, but not in the cortex, of the probiotics group compared with the vehicle group (Fig. 2A). Next, we also performed Thioflavin $\mathrm{T}$ (ThT) fluorescence staining which is used to quantify the formation of $A \beta$ fibrils to examine whether B. breve MCC1274 supplementation suppresses $A \beta$ fibril formation. We found that the percentage of ThT-positive plaque burdens area was significantly lower in the hippocampus of the probiotics group than the vehicle group (Fig. 2B). Also, both hippocampal soluble and insoluble $A \beta_{40}$ and $A \beta_{42}$ levels were significantly lower in the probiotics group than in the vehicle group (Fig. 2C, D). On the contrary, there was no significant difference in the cortical $A \beta$ deposition, $A \beta$ fibril formation as well as soluble and insoluble $A \beta_{40}$ and $A \beta_{42}$ levels between the probiotic and vehicle groups (Fig. 2A-D). Taken together, our findings suggest that $B$. breve MCC1274 supplementation reduces $A \beta$ production, deposition, and fibril formation in the hippocampus. Based on these observations, we propose that the decrease in $A \beta$ levels in the hippocampus by $B$. breve MCC1274 supplementation may prevent the memory impairment seen in $A p p^{N L-G-F}$ mice since the hippocampus is the primary area of the brain responsible for memory and learning.

\section{B. breve MCC1274 supplementation enhances the hippocampal protein level of ADAM10 in App ${ }^{N L-G-F}$ mice}

To provide further insights into the mechanisms by which $B$. breve MCC1274 supplementation decreased $A \beta$ production in the hippocampus, we measured the protein levels of $A \beta P P$ and its cleavage enzymes, namely: ADAM10 ( $\alpha$-secretase), PS1 (a component of $\gamma$-secretase), and BACE1 ( $\beta$-secretase) in the hippocampus and cortex of mouse brains by western blotting. The results revealed that $B$. breve MCC1274 supplementation significantly upregulated ADAM10 and PS1 in the hippocampus, whereas $\mathrm{A} \beta \mathrm{PP}$ and BACE1 levels did not change (Fig. 3A). However, in the cortex, there was no significant difference in the levels of A $\beta P P, A D A M 10, B A C E 1$, and PS1 between the two groups (Supplementary Figure $2 \mathrm{~A}$ ). We further evaluated the protein levels of cortical and hippocampal A $\beta P P$ fragments, including soluble (s) $\mathrm{A} \beta \mathrm{PP} \alpha$ and $\mathrm{sA} \beta \mathrm{PP} \beta$ by western blotting. A $\beta P P$ can be cleaved by ADAM10 to generate $s A \beta P P \alpha$. As expected, $s A \beta P P \alpha$ levels in the hippocampus were significantly higher in the probiotic group than in the vehicle group (Fig. 3A), whereas there was no significant difference in the cortex between the two groups (Supplementary Figure 2A). Also, both the cortical and hippocampal sA $\beta P P \beta$ levels were not significantly different between the two groups (Fig. 3A and Supplementary Figure 2A). These findings indicate that $B$. breve MCC1274 supplementation inhibits $A \beta$ production in the hippocampus through the increase in ADAM10.

\section{B. breve MCC1274 supplementation improves post-transcriptional regulation of ADAM1O}

It has been reported that hypoxia-inducible factor (HIF)- $1 \alpha$-binding sites are essential for the transcriptional activity of the ADAM10 promoter [35]. We sought to determine whether $B$. breve MCC1274 supplementation increases the ADAM10 protein level via HIF- $1 \alpha$. We found that the hippocampal HIF- $1 \alpha$ protein level (Fig. 3B), but not the cortical HIF-1 $\alpha$ protein level (Supplementary Figure 2B), was significantly higher in the probiotic group than in the vehicle group. Since the MEK/ERK signaling pathway is a key regulator of HIF-1 $\alpha$ [36], we further assessed 
A

\section{$A \beta$ staining}

Vehicle

Probiotics
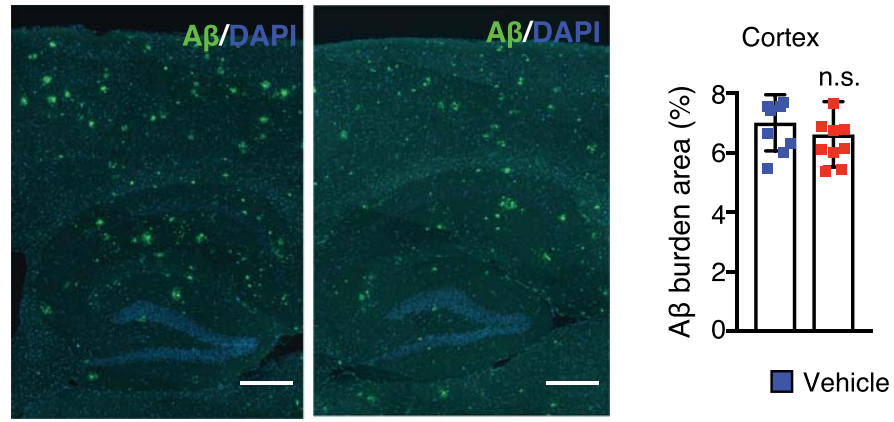

Hippocampus

B

Thioflavin T (ThT) staining

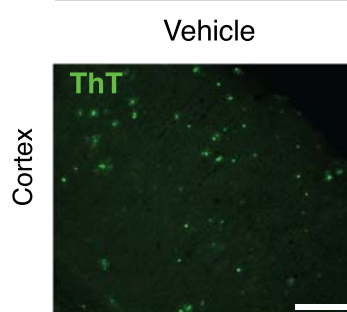

\section{Probiotics}
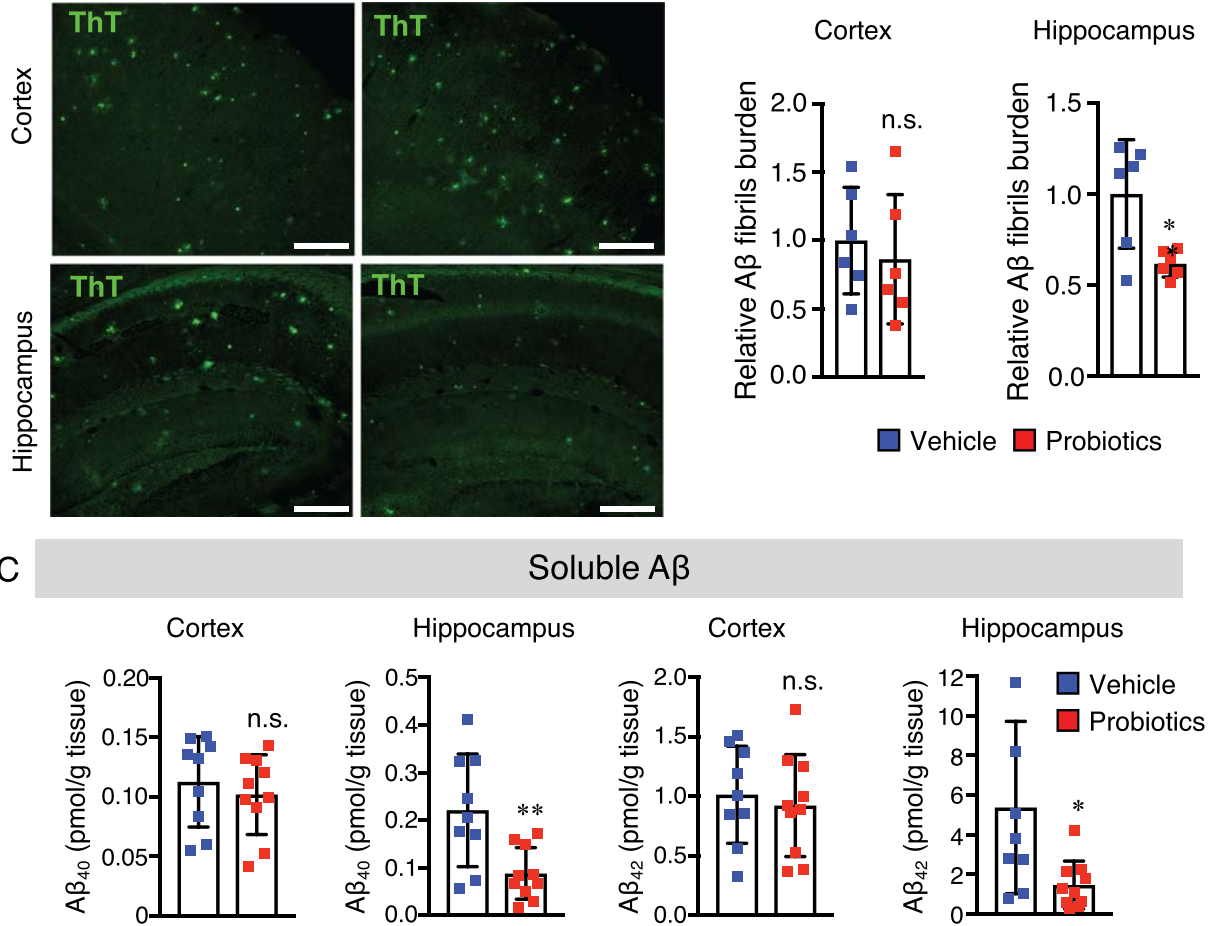

D

Insoluble $A \beta$

Cortex

Hippocampus

Cortex

Hippocampus
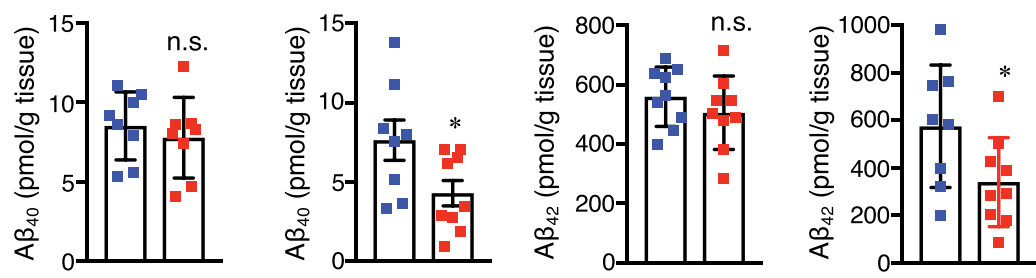

Vehicle $\square$ Probiotics

Fig. 2. (Continued) 
Fig. 2. B. breve MCC1274 supplementation reduces $\mathrm{A} \beta$ plaque burden, $\mathrm{A} \beta$ levels, and $\mathrm{A} \beta$ fibrils in the hippocampus of $A p p^{N L-G-F}$ mice. A) The representative fluorescent images of $A \beta$ plaque burden detected by anti-A $\beta$ antibody (82E1), which recognizes both $A \beta_{40}$ and $A \beta_{42}$ (left panel). $A \beta$ burden areas including the cortex and hippocampus were quantified as the percentage of immunostained area divided by all cortical and hippocampal areas (right panel). Scale bars are $250 \mu \mathrm{m} . \mathrm{B}$ ) The representative fluorescent images of A $\beta$ fibril were detected by thioflavin T (left panel). Relative A $\beta$ fibril burden in both cortex and hippocampus were quantified (right panel), Scale bars are $100 \mu \mathrm{m}$. Sandwich ELISA result of cortical and hippocampal levels of soluble $\mathrm{A} \beta_{40}$ and $\mathrm{A} \beta_{42}(\mathrm{C})$ and insoluble $\mathrm{A} \beta_{40}$ and $\mathrm{A} \beta_{42}(\mathrm{D})$ of $A p p^{N L-G-F}$ mice. $\mathrm{A} \beta$ levels were normalized to each tissue weight. Data are expressed as the mean $\pm \mathrm{SD}, n=9-10,{ }^{*} p<0.05,{ }^{* *} p<0.01$ compared with the vehicle group, as determined by Student's $t$-test.

whether B. breve MCC1274 supplementation could modulate ERK activation. Cortical and hippocampal ERK phosphorylation was assessed by western blotting. Although the total ERK level was unchanged in both the hippocampus (Fig. 3B) and cortex (Supplementary Figure 2B) between the two groups, the phosphorylated (p-) ERK level in the hippocampus (Fig. 3B), but not in the cortex (Supplementary Figure $2 \mathrm{~B}$ ), was higher in the probiotic group than in the vehicle group. Since protein kinase C (PKC) plays a significant role in ERK phosphorylation [37], we, therefore, assessed the cortical and hippocampal levels of p-PKC by western blotting. The level of hippocampal p-PKC was significantly higher in the probiotic group than in the vehicle group (Fig. 3B), whereas there were no significant differences in cortical p-PKC level between the two groups (Supplementary Figure 2B). These findings suggest that $B$. breve MCC 1274 supplementation increases the HIF- $1 \alpha$ level through the PKC-ERK pathway, consequently inducing the upregulation of the ADAM10. Next, to assess if B. breve MCC1274 supplementation altered transcription of ADAM10, we assayed the mRNA expression of ADAM10 by qRT-PCR. Although the ADAM10 mRNA expression level was not statistically significant, it tended to be increased in the hippocampus of the probiotic group compared with that of the vehicle group (Supplementary Figure 3), indicating that some unknown factor(s) might be required for the transcription of ADAM10. Therefore, we suggest that the effect of $B$. breve MCC1274 on the increased ADAM10 protein level is likely to be at the post-transcription level, including its stabilization.

\section{B. breve MCC1274 supplementation does not alter the phosphorylation of tau}

The tau hyperphosphorylation is a distinctive pathological feature in the brain of AD patients. Excessive tau phosphorylation at Thr23 (AT-180) and Ser396/Ser404 (PHF1) has been found in AD patients' brains, which inhibits physiological tau binding to microtubules, resulting in memory impair- ment [38]. Thus, we evaluated whether $B$. breve MCC1274 supplementation can affect tau phosphorylation. There was no significant alteration in total tau, AT-180 (p-Thr23), and PHF1 (p-Ser396/Ser404) levels in both the hippocampus (Fig. 4A) and cortex (Supplementary Figure 4A) between the two groups as determined by western blotting. These findings indicate that tau phosphorylation was not involved in improving cognitive function after $B$. breve MCC1274 supplementation.

\section{B. breve MCC1274 supplementation attenuates microglial activation in the hippocampus}

Our previous study suggested that oral supplementation of B. breve MCC1274 reduced inflammatory gene expression in the hippocampus [15]. However, its effect on glial activation in the brain was not explored in that study. Thus, we investigated whether oral supplementation of B. breve MCC1274 can alter glial activation. Brain sections were stained with the anti-A $\beta$ with either the anti-GFAP (an astrocytic marker) or anti-Ibal (a microglia marker) antibodies. The number of hippocampal Iba1-positive cells was significantly lower in the probiotic group than in the vehicle group (Fig. 4B), whereas, in the cortex, there was no significant difference between both groups (Supplementary Figure 4B). However, in both the hippocampus and cortex, there were no significant differences in the GFAP-positive cell number between both groups (Supplementary Figure 5). To confirm these results, we further assessed GFAP and Iba1 protein levels by western blotting, and similar results were obtained; the hippocampal Iba1 protein level was significantly lower in the probiotic group than in the vehicle group (Fig. 5A), whereas there were no significant differences in the Iba1 protein level in the cortex between both groups (Supplementary Figure 6). The GFAP level was also similar in both the hippocampus and cortex between both groups (Fig. 5A and Supplementary Figure 6). We further investigated the mRNA expression levels of the proinflammatory (IL-1 $\beta$ and IL-6) and antiinflammatory (TGF- $\beta 1$ ) cytokines that are produced 
A

Hippocampus
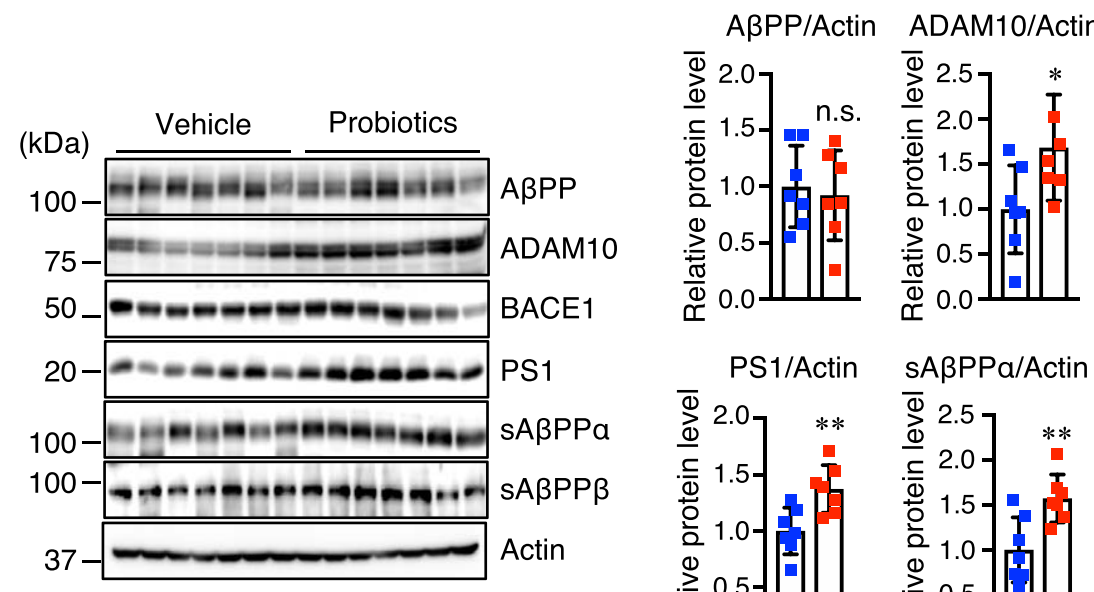

BACE1/Actin
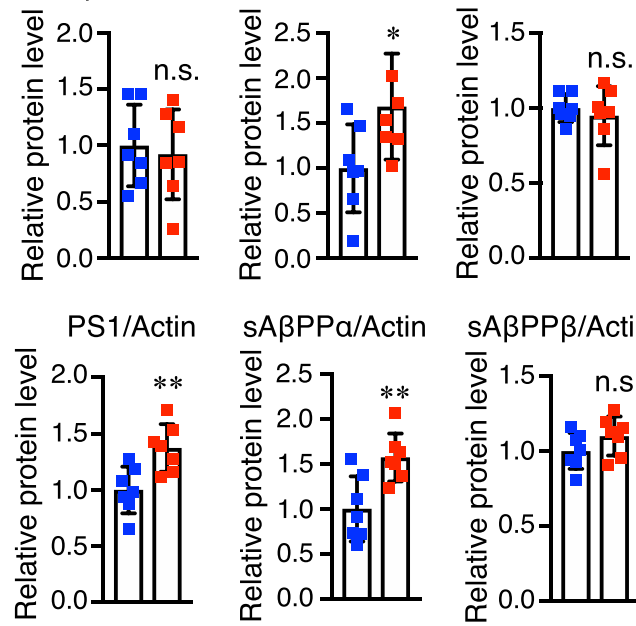

Vehicle $\square$ Probiotics

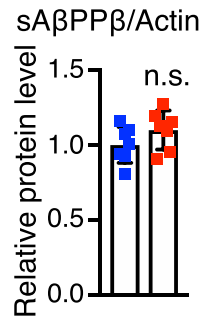

$\mathrm{B}$ Hippocampus

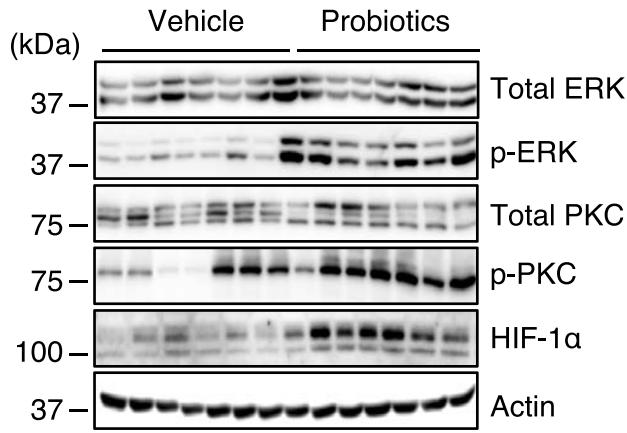

Total ERK/Actin p-ERK/Total ERK
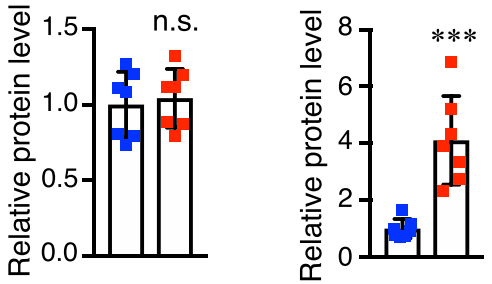

p-PKC/Total PKC

HIF-1a/Actin
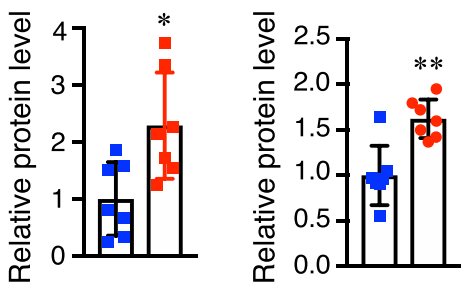

Vehicle $\square$ Probiotics

Fig. 3. B. breve MCC1274 supplementation upregulates ADAM10 protein level in the hippocampus of $A p p^{N L-G-F}$ mice. Protein levels of A $\beta P P, A D A M 10, B A C E 1, P S 1, s A \beta P P \alpha, s A \beta P P \beta$, and actin (A) and protein levels of total ERK, phosphorylated (p) ERK, total PKC, pPKC, HIF-1 $\alpha$, and actin (B) in the hippocampus were determined by western blot analysis, quantified by densitometry, normalized to actin level, and expressed as a value relative to the control. Data are expressed as the mean $\pm \mathrm{SD}, n=7 .{ }^{*} p<0.05,{ }^{* *} p<0.01,{ }^{* * *} p<0.001$ compared with the vehicle group, n.s., no significant difference, as determined by Student's $t$-test.

by microglia and astrocytes, in the hippocampal and cortical tissues by qRT-PCR analysis. Interestingly, the mRNA expression levels of IL-1 $\beta$ and IL-6 in both the hippocampus and cortex were significantly lower in the probiotic group than in the vehicle group and TGF- $\beta 1$ mRNA expression level in the hippocampus, but not in the cortex, was higher in the probiotic group than in the vehicle group, (Fig. 5B, 


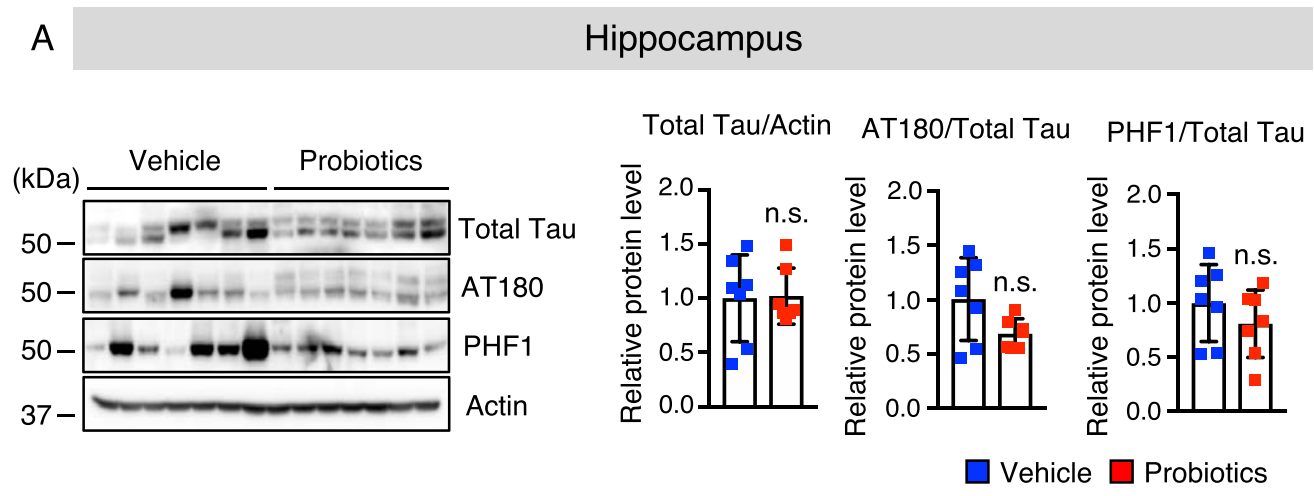

B

Hippocampus
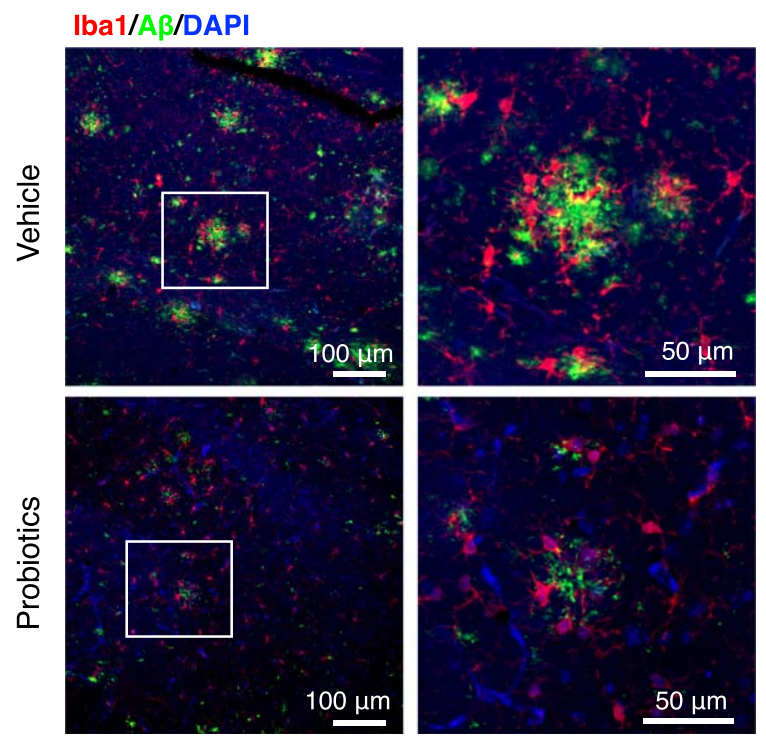

Vehicle

Probiotics

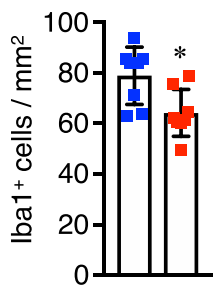

Fig. 4. B. breve MCC1274 supplementation does not affect the phosphorylation of tau and attenuates microglial activation in the hippocampus of App ${ }^{N L-G-F}$ mice. A) The protein levels of total Tau, AT180 (p-Thr23), PHF1 (p-Ser369/Ser404), and actin in the hippocampus were determined by western blot analysis, quantified by densitometry, normalized to actin level, and expressed as a value relative to the control. B) Brain sections were stained with the anti-Iba1 (red) and anti-A $\beta$ (green) antibodies, and cell nuclei were stained with DAPI (blue). Representative images of the hippocampus (left panel). Highly magnified images of the squared region in the left panels are shown in the adjacent right panels. Numbers of Iba1-positive cells (right panel) in the hippocampus. Data are expressed as the mean $\pm \mathrm{SD}, n=6-7$, ${ }^{*} p<0.05$ compared with the vehicle group, n.s., no significant difference as determined by Student's $t$-test.

C). These findings indicate that aside from microglial activation, other unknown factors altered by $B$. breve MCC1274 supplementation may be involved in the decrease in cortical IL-6 and IL-1 $\beta$ expression levels. Taken together, these findings suggest that $B$. breve MCC1274 supplementation has an anti-inflammatory function by attenuating microglial activation, and thereby decreasing the expression levels of proinflammatory cytokines as well as increasing the expression levels of anti-inflammatory cytokine.

\section{B. breve MCC1274 supplementation increases the synaptic protein levels in the hippocampus}

We also evaluated the effect of B. breve MCC1274 supplementation on synapses in the hippocampus and cortex using the anti-synaptotagmin (SYT, presynaptic protein) and anti-PSD95 (postsynaptic protein) antibodies by western blot analysis. The results of this analysis showed that the protein levels of SYT and PSD95 in the hippocampus were significantly 

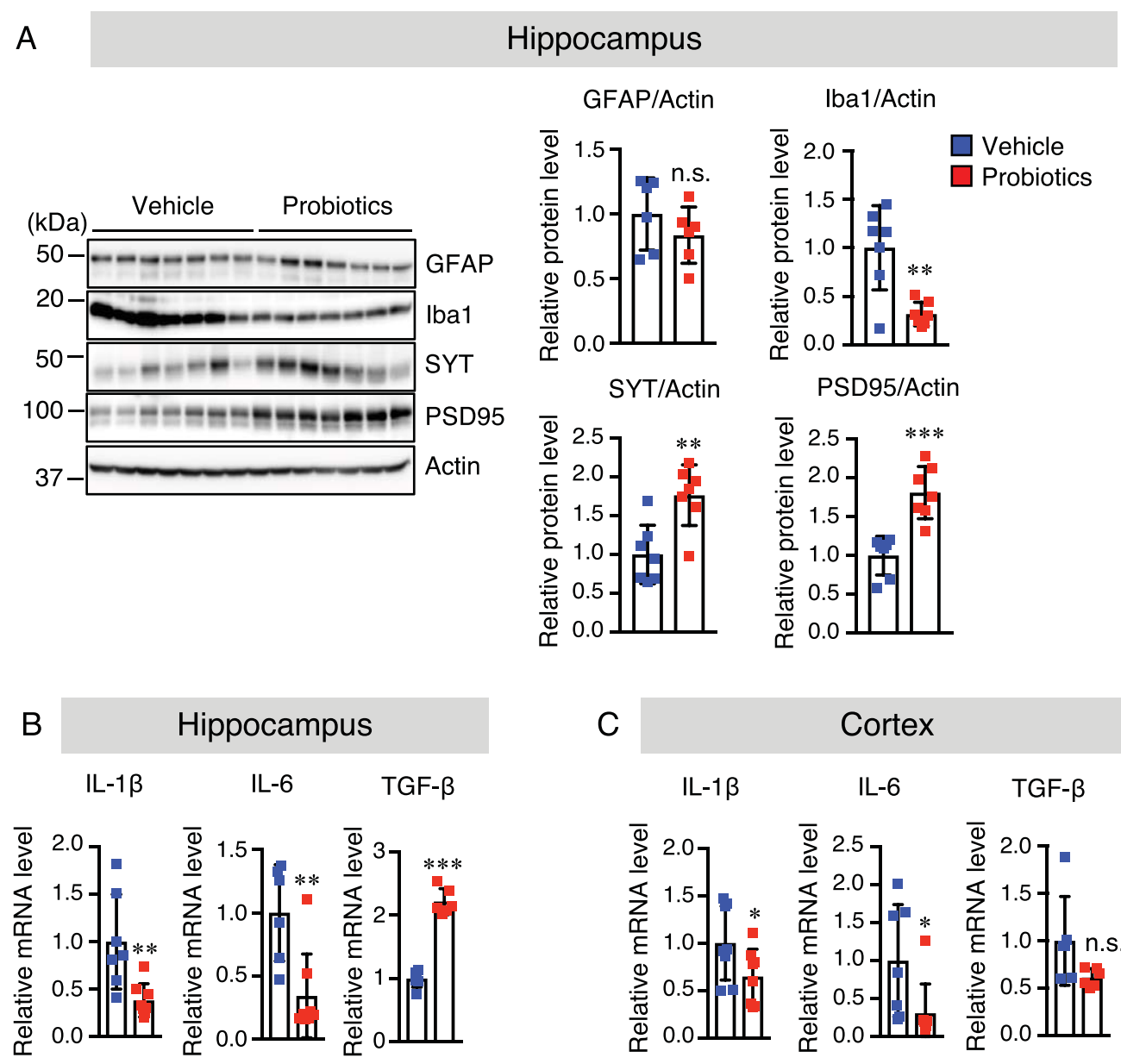

Vehicle $\square$ Probiotics

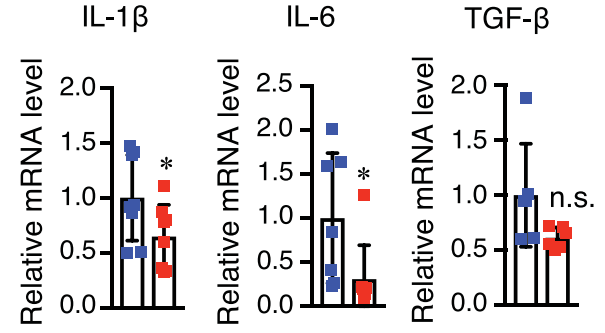

Vehicle $\square$ Probiotics
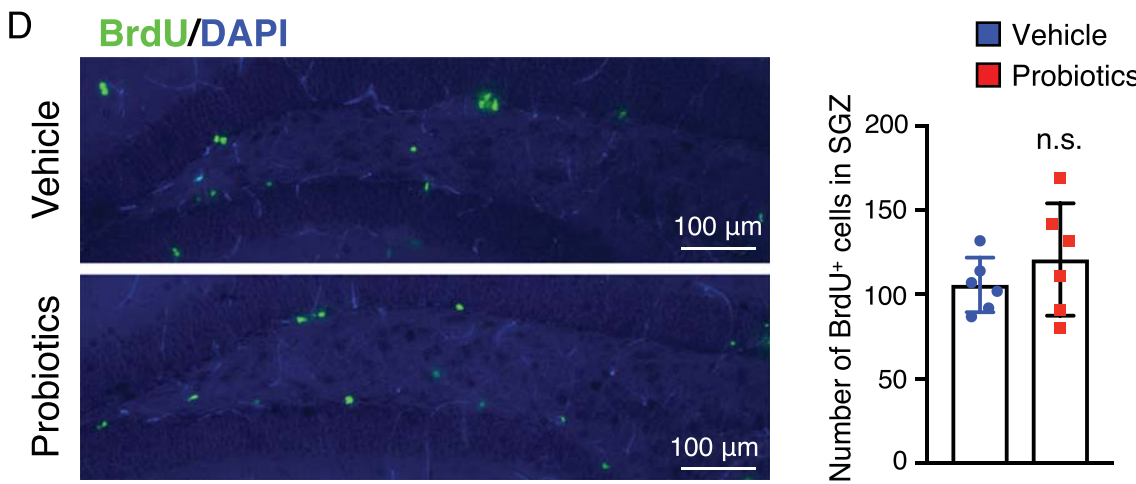

Fig. 5. B. breve MCC1274 supplementation decreases the Iba1 protein level and increases synaptic protein levels in the hippocampus, whereas it has no effect on cell proliferation in the DG of the hippocampus. A) Protein levels of GFAP, Iba1, SYT, PSD95, and actin in the hippocampus were determined by western blot analysis and quantified by densitometry, normalized to actin level, and expressed as a value relative to the control. IL- 6 and IL- $1 \beta$, and TFG- $\beta 1$ mRNA expression levels in the hippocampus (B) and cortex (C) were assessed by qRT-PCR analysis. Each mRNA expression was normalized to the corresponding amount of GAPDH mRNA. D) Brain sections were stained with the anti-BrdU antibody (green) and cell nuclei were stained with DAPI (blue) in DG in the hippocampus (left panel). The number of $\mathrm{BrdU}^{+}$cells in the SGZ of DG (right panel). Data are expressed as the mean $\pm \mathrm{SD}, n=6-7,{ }^{*} p<0.05,{ }^{* *} p<0.01,{ }^{* * *} p<0.001$ compared with the vehicle group n.s., no significant difference as determined by Student's $t$-test. 
Table 1

Effects of $B$. breve MCC1274 supplementation on gut microbiota at the genus level. There was no significant difference in composition of the gut microbiota at the genus level between $A p p^{N L-G-F}$ mice with and without $B$. breve MCC1274 supplementation as determined by Welch's $t$-test

\begin{tabular}{|c|c|c|c|c|c|c|c|c|}
\hline & & & & & & $\begin{array}{c}\text { Probiotics } \\
(\%)\end{array}$ & $\begin{array}{c}\text { Vehicle } \\
(\%)\end{array}$ & $p$ \\
\hline $\mathrm{k} \_$Bacteria & p_Actinobacteria & c_Actinobacteria & o_Bifidobacteriales & f_Bifidobacteriaceae & g_Bifidobacterium & 0.35 & 0.78 & 0.212 \\
\hline $\mathrm{k} \_$Bacteria & p_Bacteroidetes & c_Bacteroidia & o_Bacteroidales & f_Bacteroidaceae & g_Bacteroides & 2.32 & 2.32 & 0.997 \\
\hline k_Bacteria & p_Bacteroidetes & c_Bacteroidia & o_Bacteroidales & f_Porphyromonadaceae & g_Parabacteroides & 0.56 & 0.58 & 0.903 \\
\hline k_Bacteria & p_Bacteroidetes & c_Bacteroidia & o_Bacteroidales & f_Prevotellaceae & g_Prevotella & 2.91 & 2.36 & 0.485 \\
\hline k_Bacteria & p_Bacteroidetes & c_Bacteroidia & o_Bacteroidales & f_Rikenellaceae & $g_{-}$ & 1.40 & 1.55 & 0.650 \\
\hline $\mathrm{k} \_$Bacteria & p_Bacteroidetes & c_Bacteroidia & o_Bacteroidales & f_S $24-7$ & $\mathrm{~g}_{-}$ & 34.23 & 31.71 & 0.490 \\
\hline $\mathrm{k} \_$Bacteria & p_Bacteroidetes & c_Bacteroidia & o_Bacteroidales & f_[Paraprevotellaceae] & g_[Prevotella] & 7.81 & 7.48 & 0.870 \\
\hline $\mathrm{k} \_$Bacteria & p_Firmicutes & c_Bacilli & o_Lactobacillales & f_Lactobacillaceae & g_Lactobacillus & 10.51 & 13.99 & 0.339 \\
\hline k_Bacteria & p_Firmicutes & c_Bacilli & o_Turicibacterales & f_Turicibacteraceae & g_Turicibacter & 4.12 & 5.13 & 0.656 \\
\hline k_Bacteria & p_Firmicutes & c_Clostridia & o_Clostridiales & - & - & 3.35 & 4.20 & 0.444 \\
\hline k_Bacteria & p_Firmicutes & c_Clostridia & o_Clostridiales & $\mathrm{f}_{-}$ & $g_{-}$ & 4.49 & 3.43 & 0.304 \\
\hline k_Bacteria & p_Firmicutes & c_Clostridia & o_Clostridiales & f_Lachnospiraceae & - & 9.58 & 6.51 & 0.270 \\
\hline k_Bacteria & p_Firmicutes & c_Clostridia & o_Clostridiales & f_Lachnospiraceae & $\mathrm{g}_{-}$ & 0.62 & 0.75 & 0.710 \\
\hline k_Bacteria & p_Firmicutes & c_Clostridia & o_Clostridiales & f_Lachnospiraceae & g_Coprococcus & 2.01 & 1.54 & 0.328 \\
\hline k_Bacteria & p_Firmicutes & c_Clostridia & o_Clostridiales & f_Lachnospiraceae & g_[Ruminococcus] & 0.78 & 0.53 & 0.245 \\
\hline k_Bacteria & p_Firmicutes & c_Clostridia & o_Clostridiales & f_Ruminococcaceae & g_Oscillospira & 1.26 & 0.75 & 0.060 \\
\hline k_Bacteria & p_Firmicutes & c_Clostridia & o_Clostridiales & f_Ruminococcaceae & g_Ruminococcus & 1.73 & 1.25 & 0.170 \\
\hline k_Bacteria & p_Firmicutes & c_Erysipelotrichi & o_Erysipelotrichales & f_Erysipelotrichaceae & g_Allobaculum & 5.07 & 8.24 & 0.288 \\
\hline
\end{tabular}

higher in the probiotic group than in the vehicle group (Fig. 5A), whereas there were no significant differences in their protein levels in the cortex between both groups (Supplementary Figure 6). These findings suggest that $B$. breve MCC1274 supplementation increases synaptic density in the hippocampus.

\section{B. breve MCC1274 supplementation does not affect cell proliferation in the subgranular zone of the dentate gyrus}

To examine the effect of $B$. breve MCC1274 supplementation on the cell proliferation in the subgranular zone (SGZ) of the dentate gyrus (DG) of the hippocampus, $A p p^{N L-G-F}$ mice were injected with BrdU. The number of BrdU-positive cells was calculated in this area for both the probiotic and vehicle groups. Although the difference in the BrdU-positive cell number in the SGZ of the DG area was not statistically significant, they tended to be higher in the probiotic group than in the vehicle group (Fig. 5D).

\section{B. breve MCC1274 supplementation does not change gut microbiota composition}

Fecal samples were analyzed to investigate whether $B$. breve MCC1274 supplementation caused any changes in the gut microbiota composition. PCoA based on weighted unifrac distance showed no intergroup difference (Fig. 6). Furthermore, in

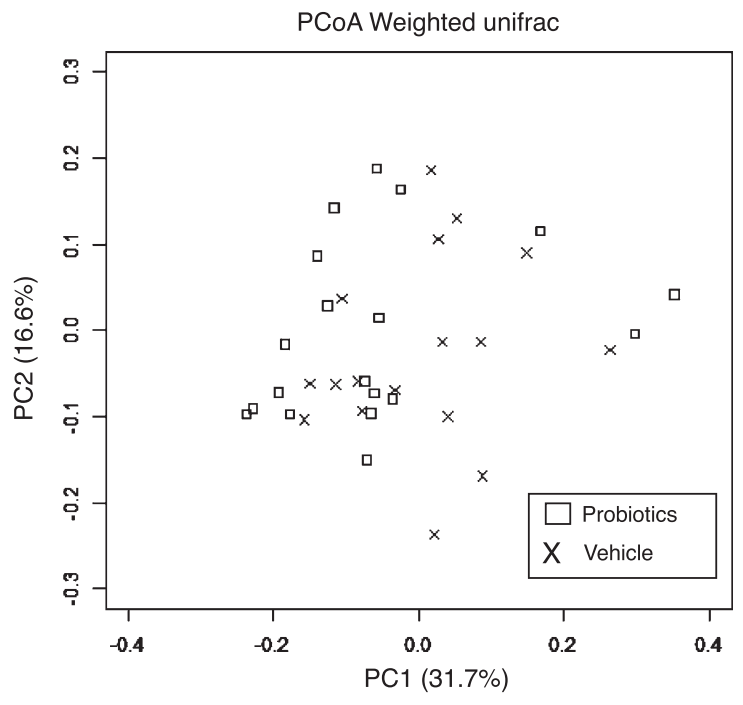

Fig. 6. Effects of B. breve MCC1274 supplementation on gut microbiota. PCoA based on weighted unifrac distance from $16 \mathrm{~S}$ rRNA sequencing data did not show a significant difference between $A p p^{N L-G-F}$ mice with and without $B$. breve MCC1274 supplementation as determined by permutation MANOVA ( $p=$ $0.379)$

the analysis at the genus level, no change in the gut microbiota composition caused by the administration of probiotics was observed (Table 1). These results are consistent with our previous finding that B. breve MCC1274 supplementation does not affect gut microbiota in ddY mice induced by intracerebroventricular injection with $\mathrm{A} \beta$ [15]. 


\section{DISCUSSION}

Several studies provided strong evidence for the link between the gut microbiota and the brain through the microbiota-gut-brain axis. Therefore, any disruption or alteration in an individual's gut microbiota can affect the individual's behavior and cause psychiatric and neurodegenerative diseases, including AD. Recently, we have demonstrated that orally administered B. breve A1 (MCC1274) prevented cognitive deficits in $\mathrm{A} \beta$-injected mice through the suppression of hippocampal inflammation and immunoreactive genes [15] and that providing the same probiotic to MCI subjects for 16 weeks improved their cognitive function [23]. However, the underlying molecular mechanism by which probiotic supplementation prevents cognitive deficits in humans and animals has not been fully elucidated. In this study, therefore, we further investigated the role of $B$. breve MCC1274 in AD-like pathologies to evaluate its ability to alter $\mathrm{A} \beta$ production and glial activation in $A p p^{N L-G-F}$ mice. Here, we demonstrated that $B$. breve MCC 1274 supplementation significantly prevented memory impairment and found decreased soluble and insoluble $A \beta$ levels as well as the $A \beta$ plaque load in the hippocampus. We also revealed that $B$. breve MCC1274 supplementation attenuated microglial activation accompanied by the reduction in IL-1 $\beta$ and IL-6 mRNA expression levels as well as the increases in TGF- $\beta 1$ mRNA expression levels and synaptic protein levels.

Several transgenic mouse models overexpressing human $\mathrm{A} \beta \mathrm{PP}$ have been generated for use in the investigation of AD-like pathologies and drug treatments for AD. However, these mouse models overexpress $A \beta P P$ and $A \beta P P$ fragments such as sA $\beta P P, C T F \alpha$, and $C T F \beta$, which may possess some biological functions and cause the expression of artefactual phenotypes. Recently, App ${ }^{N L-G-F}$ mice carrying Swedish, Beyreuther/Iberian, and Arctic mutations associated with familial AD have been generated to overcome these problems. Since these mice showed cognitive impairment, $A \beta$ amyloidosis, and neuroinflammation without physiological A $\beta P P$ overexpression, we, therefore, used this mouse model in this study.

It has been demonstrated that the supplementation of some probiotics such as Bifidobacterium longum and Lactobacillus acidophilus significantly improved the cognitive function in $A \beta$-treated rats and $A \beta P P / P S 1$ transgenic mice $[39,40]$. We also previously demonstrated that $B$. breve $\mathrm{MCC} 1274$ supplementation ameliorated the cognitive decline in A $\beta$-injected mice [15] and improved the cognitive function in MCI subjects [23]. In this study, we conducted the NOR test to evaluate short-term memory and recognition ability and found that $A p p^{N L-G-F}$ mice treated with saline showed short-term memory impairment at the age of seven months, which is consistent with a previous report [24]. However, the mice supplemented with $B$. breve MCC1274 did not show memory impairment, suggesting that $B$. breve MCC1274 supplementation might prevent cognitive impairment in $\mathrm{AD}$.

There is strong evidence showing that the accumulation of $A \beta$ in the brain can induce memory impairment. Therefore, reducing $A \beta$ levels and thereby preventing $A \beta$ toxicity in the brain are thought to be preventive therapy for $\mathrm{AD}$ progression. It has been reported that some probiotics such as Bifidobacterium longum, Lactobacillus acidophilus, and $L$. plantarum supplemented with memantine decreased the $A \beta$ levels in the brain of $A \beta P P / P S 1$ transgenic mice $[40,41]$. Here, we also found that $B$. breve MCC1274 supplementation significantly decreased hippocampal $\mathrm{A} \beta$ production and $\mathrm{A} \beta$ plaque load of $A p p^{N L-G-F}$ mice, indicating that this probiotic may protect the hippocampus against the toxic effect of $A \beta$ and thus prevent short-term memory impairment.

Although some probiotics can reduce $A \beta$ levels in the brain, the precise mechanism by which these probiotics decrease $A \beta$ levels has not been completely clarified. Here, we found that $B$. breve MCC1274 supplementation increased the hippocampal ADAM10 protein level associated with the increased $\operatorname{sA} \beta P P \alpha$ and CTF $\alpha$ levels, leading to decreased A $\beta$ levels in the hippocampus. Thus, our findings suggest that B. breve MCC1274 supplementation may contribute to A $\beta P P$ metabolism toward the non-amyloidogenic pathway through the upregulation of the hippocampal ADAM10 protein level, resulting in the decrease in hippocampal $\mathrm{A} \beta$ production and deposition.

The PKC-ERK-HIF- $1 \alpha$ signaling pathway has been involved in ADAM10 transcription. Here, we found that although B. breve MCC1274 supplementation activated PKC and ERK leading to the increased ADAM10 protein level, we observed no increase in the ADAM10 mRNA expression level, indicating that some unknown factor(s) might be required for the transcription of ADAM10. Therefore, we suggest that the effect of B. breve MCC1274 on the increased ADAM10 protein level is likely to be at the posttranscription level, including its stabilization. Further 
investigation is required to explore which factor(s) are involved in ADAM10 transcription.

Since previous studies have shown that the improvement of synaptic activity increased ADAM 10 levels [42, 43], the increase in synaptic density by $B$. breve MCC1274 supplementation might also be involved in the increase in ADAM10 level. Furthermore, the enhancement of ERK activation, particularly in the hippocampus, is necessary for the increase in synaptic protein levels and memory formation [44]. Here, we found that B. breve MCC1274 supplementation markedly increased both presynaptic (SYT) and postsynaptic (PSD95) protein levels and enhanced ERK activation in the hippocampus. Taken together, the enhancement of ERK activation by $B$. breve MCC1274 supplementation may play a role in the increase in synaptic density and subsequently an increase in the ADAM10 level as well as memory formation.

The effect of $B$. breve MCC1274 on tau phosphorylation is unknown. Thus, we also examined whether B. breve MCC1274 supplementation can affect tau phosphorylation. Hyperphosphorylated tau at AT180 and PHF1 epitopes has been indicated to suppress its binding to microtubules, thereby diminishing its function [38]. Our results demonstrated that $B$. breve MCC1274 supplementation could not alter tau phosphorylation at AT180 and PHF1 epitopes in both the cortex and hippocampus. Therefore, the improvement of brain performance induced by B. breve MCC1274 supplementation is not associated with tau phosphorylation.

Activated microglia and astrocytes are considered pathological hallmarks of $\mathrm{AD}$ as they induce both $\mathrm{A} \beta$ deposition and release of proinflammatory cytokines such as TNF $\alpha$, IL-1 $\beta$, and IL-6 [5, $45,46]$. It has been shown that IL- $1 \beta$ and IL-6 play roles in regulating cognitive function; blocking IL-1 $\beta$ in $\mathrm{AD}$ mouse models protects against cognitive deficits [47], and the upregulation of IL-6 contributes to cognitive deficits in humans [48]. Several studies have shown that some probiotic strains appeared to have an anti-inflammatory effect, which effectively attenuated cognitive dysfunction $[49,50]$. We also previously demonstrated that oral $B$. breve MCC1274 supplementation suppressed the inflammation in the hippocampus of $\mathrm{A} \beta$-injected mice. Here, we demonstrated that B. breve MCC1274 supplementation significantly decreased both the number of $\mathrm{Iba}^{+}$cells surrounding $A \beta$ plaques and the Ibal protein level in the hippocampus of $A p p^{N L-G-F}$ mice. It has been reported that there are two major phenotypes of activated microglia. M1 and M2, which secrete pro-inflammatory and anti-inflammatory cytokines, respectively. In this study, B. breve MCC1274 supplementation decreased the mRNA expression levels of pro-inflammatory cytokines (IL-6 and IL-1 $\beta$ ) and increased that of an anti-inflammatory cytokine (TGF- $\beta 1$ ) in the hippocampus. These results suggest that $B$. breve MCC1274 supplementation might convert M1 to M2 phenotype and that the altered levels of proand anti-inflammatory cytokines could be derived from microglia because the number of $\mathrm{GFAP}^{+}$ cells and GFAP protein levels were not altered by $B$. breve MCC1274 supplementation. Taken together, our results suggest that $B$. breve $\mathrm{MCC} 1274$ supplementation may shift activated microglia from a pro-inflammatory M1 to an anti-inflammatory M2 phenotype. Further investigation is required to test this hypothesis using specific microglia phenotype markers.

Our findings showed that IL- 6 and IL- $1 \beta$ mRNA expression levels were decreased in both the cortex and hippocampus of mice given B. breve $\mathrm{MCC} 1274$ supplementation; the reduction in proinflammatory cytokine expression levels in the hippocampus may be due to the attenuation of microglia activation, whereas that in the cortex may be a result of the reduction of systemic anti-inflammatory cytokine release. Previous animal and human studies showed that probiotics have an intestinal anti-inflammatory function that leads to the reduction in intestinal IL-1 $\beta$ and IL-6 levels [51-53]. Moreover, ingested probiotics plays a systemic anti-inflammatory role and decrease the levels of proinflammatory cytokines released in serum, such as IL-6 and IL-1 $\beta$ [54]. Thus, $B$. breve MCC1274 supplementation might reduce the inflammatory reaction in the brain of $A p p^{N L-G-F}$ mice, at least in part, by attenuating microglial activation.

Interestingly, the effects of $B$. breve MCC1274 on $\mathrm{A} \beta$ pathology, microglial activation, and synaptic protein levels were found only in the hippocampus and not in the cortex in this study. Although the reason for this is unclear, one possibility is that probiotics can activate specific brain regions through the vagus afferent nerve that is involved in the communication of the microbiome-gut-brain axis [55]. For example, the ingestion of a Lactobacillus strain upregulated the mRNA expression of GABA receptors in a regionaldependent manner through the vagus nerve, which could be stimulated by the probiotics. Further studies are necessary to elucidate the potential mechanisms 
associated with the effects of B. breve MCC1274 on the hippocampus.

Reduction of hippocampal neurogenesis has been reported in $\mathrm{AD}$ cases of both human and AD-like mouse models. A previous study showed that $L$. helveticus R0052 and B. longum R0175 prevent the alteration of neurogenesis caused by chronic stress [56], and it has been reported that VSL\#3 can reverse the impairment of neurogenesis that occurred as a result of antibiotics treatment [57]. Therefore, we examined whether B. breve MCC1274 supplementation can affect cell proliferation in the hippocampus of $A p p^{N L-G-F}$ mice. In this study, we found that $\mathrm{BrdU}^{+}$cell number did not significantly differ between the probiotic and vehicle groups. The difference between our findings and the previous findings may be due to the differences in the probiotic strain and mouse type used. Thus, B. breve MCC1274 supplementation is not effective in improving hippocampal neurogenesis in $A p p^{N L-G-F}$ mice.

In this study, we did not observe a marked change in the gut microbiota following the administration of $B$. breve MCC1274, which is consistent with our previous finding [15], implying that another mechanism(s) might exist for ameliorating AD-like pathologies. Our previous study has shown that viable or nonviable B. breve MCC1274 could also prevent cognitive impairment and suppress inflammatory gene expression in the hippocampus of AD-like mice, implying that some structural components of the probiotics may be involved in the modulation of neuronal immune responses [15]. Thus, one possible mechanism by which the probiotics ameliorate AD-like pathologies is that some structural components are likely to be involved in their effects. However, further investigation on whether nonviable B. breve MCC1274 has ameliorating effects on $\mathrm{AD}$ like pathologies is necessary.

In summary, we have shown that oral $B$. breve MCC1274 supplementation alleviated cognitive dysfunction and $\mathrm{A} \beta$ production and deposition in $A p p^{N L-G-F}$ mice. More importantly, we showed for the first time that oral supplementation of $B$. breve MCC1274 positively regulated the ADAM10 protein level in the hippocampus leading to the reduction of $\mathrm{A} \beta$ production. The oral $B$. breve MCC1274 supplementation also alleviated neuroinflammation in the brains of $A p p^{N L-G-F}$ mice, thereby demonstrating its neuroprotective properties. Our findings support the possibility that oral B. breve MCC 1274 supplementation might potentially prevent $\mathrm{AD}$ progression.

\section{ACKNOWLEDGMENTS}

The authors thank Professor Takaomi Saido (Laboratory for Proteolytic Neuroscience, RIKEN Brain Science Institute, Wako, Japan) and Professor Takashi Saito (Department of Neurocognitive Science, Nagoya City University Graduate School of Medical Science Nagoya, Japan) for providing APP-KI mice as a gift. We also thank Dr. Frank Bernier (Next Generation Science Institute, Morinaga Milk Industries Co., Ltd.) for critically reading our manuscript. We acknowledge the assistance of the Research Equipment Sharing Center at Nagoya City University. This work was supported by a Grant-inAid for Scientific Research B 16H05559 (to M.M.), a Grant-in-Aid for Challenging Exploratory Research 15K15712 (to M.M.), and Grant-in-Aid for Scientific Research C 20K07762 (to C-G. J.) from the Ministry of Education, Culture, Sports, Science and Technology, Japan, and by the Project of Translational and Clinical Research seed A from the Japan Agency for Medical Research and Development (AMED, A-128) (to M.M.), and the Kobayashi Foundation, Osaka, Japan (to M.M.). This work was also supported by Morinaga Milk Industry Co., Ltd.

Authors' disclosures available online (https:// www.j-alz.com/manuscript-disclosures/21-5025r2).

\section{SUPPLEMENTARY MATERIAL}

The supplementary material is available in the electronic version of this article: https://dx.doi.org/ 10.3233/JAD-215025.

\section{REFERENCES}

[1] Mount C, Downton C (2006) Alzheimer disease: progress or profit? Nat Med 12, 780-784.

[2] Takasugi N, Tomita T, Hayashi I, Tsuruoka M, Niimura M, Takahashi Y, Thinakaran G, Iwatsubo T (2003) The role of presenilin cofactors in the gamma-secretase complex. Nature 422, 438-441.

[3] Lammich S, Kojro E, Postina R, Gilbert S, Pfeiffer R, Jasionowski M, Haass C, Fahrenholz F (1999) Constitutive and regulated alpha-secretase cleavage of Alzheimer's amyloid precursor protein by a disintegrin metalloprotease. Proc Natl Acad Sci U S A 96, 3922-3927.

[4] Castro-Gomez S, Binder J, Heneka MT (2019) [Neuroinflammation as motor of Alzheimer's disease]. Nervenarzt 90, 898-906.

[5] Heneka MT, Carson MJ, El Khoury J, Landreth GE, Brosseron F, Feinstein DL, Jacobs AH, Wyss-Coray T, Vitorica J, Ransohoff RM, Herrup K, Frautschy SA, Finsen B, Brown GC, Verkhratsky A, Yamanaka K, Koistinaho J, Latz E, Halle A, Petzold GC, Town T, Morgan D, Shinohara 
ML, Perry VH, Holmes C, Bazan NG, Brooks DJ, Hunot S, Joseph B, Deigendesch N, Garaschuk O, Boddeke E, Dinarello CA, Breitner JC, Cole GM, Golenbock DT, Kummer MP (2015) Neuroinflammation in Alzheimer's disease. Lancet Neurol 14, 388-405.

[6] Ogawa M, Fukuyama H, Ouchi Y, Yamauchi H, Kimura J (1996) Altered energy metabolism in Alzheimer's disease. J Neurol Sci 139, 78-82.

[7] Jellinger KA (2003) Is Alzheimer's disease a vascular disorder? J Alzheimers Dis 5, 247-250; discussion 251-262.

[8] Belkaid Y, Hand TW (2014) Role of the microbiota in immunity and inflammation. Cell 157, 121-141.

[9] Carabotti M, Scirocco A, Maselli MA, Severi C (2015) The gut-brain axis: interactions between enteric microbiota, central and enteric nervous systems. Ann Gastroenterol 28, 203-209.

[10] Farzi A, Frohlich EE, Holzer P (2018) Gut microbiota and the neuroendocrine system. Neurotherapeutics 15, $5-22$.

[11] Gareau MG, Sherman PM, Walker WA (2010) Probiotics and the gut microbiota in intestinal health and disease. Nat Rev Gastroenterol Hepatol 7, 503-514.

[12] Cattaneo A, Cattane N, Galluzzi S, Provasi S, Lopizzo N, Festari C, Ferrari C, Guerra UP, Paghera B, Muscio C, Bianchetti A, Volta GD, Turla M, Cotelli MS, Gennuso M, Prelle A, Zanetti O, Lussignoli G, Mirabile D, Bellandi D, Gentile S, Belotti G, Villani D, Harach T, Bolmont T, Padovani A, Boccardi M, Frisoni GB, INDIA-FBP Group (2017) Association of brain amyloidosis with proinflammatory gut bacterial taxa and peripheral inflammation markers in cognitively impaired elderly. Neurobiol Aging 49, 60-68.

[13] Minter MR, Zhang C, Leone V, Ringus DL, Zhang X, Oyler-Castrillo P, Musch MW, Liao F, Ward JF, Holtzman DM, Chang EB, Tanzi RE, Sisodia SS (2016) Antibioticinduced perturbations in gut microbial diversity influences neuro-inflammation and amyloidosis in a murine model of Alzheimer's disease. Sci Rep 6, 30028.

[14] Wu SC, Cao ZS, Chang KM, Juang JL (2017) Intestinal microbial dysbiosis aggravates the progression of Alzheimer's disease in Drosophila. Nat Commun 8, 24.

[15] Kobayashi Y, Sugahara H, Shimada K, Mitsuyama E, Kuhara T, Yasuoka A, Kondo T, Abe K, Xiao JZ (2017) Therapeutic potential of Bifidobacterium breve strain A1 for preventing cognitive impairment in Alzheimer's disease. Sci Rep 7, 13510.

[16] Johnson KV, Foster KR (2018) Why does the microbiome affect behaviour? Nat Rev Microbiol 16, 647-655.

[17] Ho L, Ono K, Tsuji M, Mazzola P, Singh R, Pasinetti GM (2018) Protective roles of intestinal microbiota derived short chain fatty acids in Alzheimer's disease-type beta-amyloid neuropathological mechanisms. Expert Rev Neurother 18, 83-90.

[18] Erny D, Hrabe de Angelis AL, Jaitin D, Wieghofer P, Staszewski O, David E, Keren-Shaul H, Mahlakoiv T, Jakobshagen K, Buch T, Schwierzeck V, Utermohlen O, Chun E, Garrett WS, McCoy KD, Diefenbach A, Staeheli P, Stecher B, Amit I, Prinz M (2015) Host microbiota constantly control maturation and function of microglia in the CNS. Nat Neurosci 18, 965-977.

[19] Perry VH, Nicoll JA, Holmes C (2010) Microglia in neurodegenerative disease. Nat Rev Neurol 6, 193-201.

[20] Streit WJ, Mrak RE, Griffin WS (2004) Microglia and neuroinflammation: a pathological perspective. J Neuroinflammation 1, 14.
[21] Boutajangout A, Wisniewski T (2013) The innate immune system in Alzheimer's disease. Int J Cell Biol 2013, 576383.

[22] Hunter JM, Kwan J, Malek-Ahmadi M, Maarouf CL, Kokjohn TA, Belden C, Sabbagh MN, Beach TG, Roher AE (2012) Morphological and pathological evolution of the brain microcirculation in aging and Alzheimer's disease. PLoS One 7, e36893.

[23] Xiao J, Katsumata N, Bernier F, Ohno K, Yamauchi Y, Odamaki T, Yoshikawa K, Ito K, Kaneko T (2020) Probiotic Bifidobacterium breve in improving cognitive functions of older adults with suspected mild cognitive impairment: a randomized, double-blind, placebo-controlled trial. $J$ Alzheimers Dis 77, 139-147.

[24] Saito T, Matsuba Y, Mihira N, Takano J, Nilsson P, Itohara S, Iwata N, Saido TC (2014) Single App knock-in mouse models of Alzheimer's disease. Nat Neurosci 17, 661-663.

[25] Abdelhamid M, Jung CG, Zhou C, Abdullah M, Nakano M, Wakabayashi H, Abe F, Michikawa M (2020) Dietary lactoferrin supplementation prevents memory impairment and reduces amyloid-beta generation in $\mathrm{J} 20$ mice. $J$ Alzheimers Dis 74, 245-259.

[26] Bevins RA, Besheer J (2006) Object recognition in rats and mice: a one-trial non-matching-to-sample learning task to study 'recognition memory'. Nat Protoc 1, 1306-1311.

[27] Hosono T, Mouri A, Nishitsuji K, Jung CG, Kontani M, Tokuda H, Kawashima H, Shibata H, Suzuki T, Nabehsima T, Michikawa M (2015) Arachidonic or docosahexaenoic acid diet prevents memory impairment in $\operatorname{Tg} 2576$ mice. $J$ Alzheimers Dis 48, 149-162.

[28] Kato K, Ishida S, Tanaka M, Mitsuyama E, Xiao JZ, Odamaki T (2018) Association between functional lactase variants and a high abundance of Bifidobacterium in the gut of healthy Japanese people. PLoS One 13, e0206189.

[29] Caporaso JG, Kuczynski J, Stombaugh J, Bittinger K, Bushman FD, Costello EK, Fierer N, Pena AG, Goodrich JK, Gordon JI, Huttley GA, Kelley ST, Knights D, Koenig JE, Ley RE, Lozupone CA, McDonald D, Muegge BD, Pirrung M, Reeder J, Sevinsky JR, Turnbaugh PJ, Walters WA, Widmann J, Yatsunenko T, Zaneveld J, Knight R (2010) QIIME allows analysis of high-throughput community sequencing data. Nat Methods 7, 335-336.

[30] Kuczynski J, Stombaugh J, Walters WA, Gonzalez A, Caporaso JG, Knight R (2012) Using QIIME to analyze 16S rRNA gene sequences from microbial communities. Curr Protoc Microbiol Chapter 1, Unit 1E 5.

[31] Callahan BJ, McMurdie PJ, Rosen MJ, Han AW, Johnson AJ, Holmes SP (2016) DADA2: High-resolution sample inference from Illumina amplicon data. Nat Methods 13, 581-583.

[32] Mehla J, Lacoursiere SG, Lapointe V, McNaughton BL, Sutherland RJ, McDonald RJ, Mohajerani MH (2019) Agedependent behavioral and biochemical characterization of single APP knock-in mouse (APP(NL-G-F/NL-G-F)) model of Alzheimer's disease. Neurobiol Aging 75, 25-37.

[33] Whyte LS, Hemsley KM, Lau AA, Hassiotis S, Saito T, Saido TC, Hopwood JJ, Sargeant TJ (2018) Reduction in open field activity in the absence of memory deficits in the App(NL-G-F) knock-in mouse model of Alzheimer's disease. Behav Brain Res 336, 177-181.

[34] Latif-Hernandez A, Shah D, Craessaerts K, Saido T, Saito T, De Strooper B, Van der Linden A, D'Hooge R (2019) Subtle behavioral changes and increased prefrontalhippocampal network synchronicity in APP(NL-G-F) mice before prominent plaque deposition. Behav Brain Res 364, 431-441. 
[35] Shukla M, Htoo HH, Wintachai P, Hernandez JF, Dubois C, Postina R, Xu H, Checler F, Smith DR, Govitrapong P, Vincent B (2015) Melatonin stimulates the nonamyloidogenic processing of betaAPP through the positive transcriptional regulation of ADAM10 and ADAM17. J Pineal Res 58, 151-165.

[36] Richard DE, Berra E, Gothie E, Roux D, Pouyssegur J (1999) p42/p44 mitogen-activated protein kinases phosphorylate hypoxia-inducible factor 1alpha (HIF-1alpha) and enhance the transcriptional activity of HIF-1.J Biol Chem 274, 32631-32637.

[37] Tosini G, Owino S, Guillaume JL, Jockers R (2014) Understanding melatonin receptor pharmacology: latest insights from mouse models, and their relevance to human disease. Bioessays 36, 778-787.

[38] Gong CX, Iqbal K (2008) Hyperphosphorylation of microtubule-associated protein tau: a promising therapeutic target for Alzheimer disease. Curr Med Chem 15, 23212328.

[39] Athari Nik Azm S, Djazayeri A, Safa M, Azami K, Ahmadvand B, Sabbaghziarani F, Sharifzadeh M, Vafa M (2018) Lactobacilli and bifidobacteria ameliorate memory and learning deficits and oxidative stress in beta-amyloid (1-42) injected rats. Appl Physiol Nutr Metab 43, 718-726.

[40] Abraham D, Feher J, Scuderi GL, Szabo D, Dobolyi A, Cservenak M, Juhasz J, Ligeti B, Pongor S, Gomez-Cabrera MC, Vina J, Higuchi M, Suzuki K, Boldogh I, Radak Z (2019) Exercise and probiotics attenuate the development of Alzheimer's disease in transgenic mice: Role of microbiome. Exp Gerontol 115, 122-131.

[41] Wang QJ, Shen YE, Wang X, Fu S, Zhang X, Zhang YN, Wang RT (2020) Concomitant memantine and Lactobacillus plantarum treatment attenuates cognitive impairments in APP/PS1 mice. Aging (Albany NY) 12, 628-649.

[42] Wan XZ, Li B, Li YC, Yang XL, Zhang W, Zhong L, Tang SJ (2012) Activation of NMDA receptors upregulates a disintegrin and metalloproteinase 10 via a Wnt/MAPK signaling pathway. J Neurosci 32, 3910-3916.

[43] Marcello E, Gardoni F, Mauceri D, Romorini S, Jeromin A, Epis R, Borroni B, Cattabeni F, Sala C, Padovani A, Di Luca M (2007) Synapse-associated protein-97 mediates alphasecretase ADAM10 trafficking and promotes its activity. $J$ Neurosci 27, 1682-1691.

[44] Cao G, Zhu J, Zhong Q, Shi C, Dang Y, Han W, Liu X, Xu M, Chen T (2013) Distinct roles of methamphetamine in modulating spatial memory consolidation, retrieval, reconsolidation and the accompanying changes of ERK and CREB activation in hippocampus and prefrontal cortex. Neuropharmacology 67, 144-154.

[45] Rojo LE, Fernandez JA, Maccioni AA, Jimenez JM, Maccioni RB (2008) Neuroinflammation: implications for the pathogenesis and molecular diagnosis of Alzheimer's disease. Arch Med Res 39, 1-16.

[46] Wyss-Coray T (2006) Inflammation in Alzheimer disease: driving force, bystander or beneficial response? Nat Med 12, 1005-1015.
[47] Kitazawa M, Cheng D, Tsukamoto MR, Koike MA, Wes PD, Vasilevko V, Cribbs DH, LaFerla FM (2011) Blocking IL-1 signaling rescues cognition, attenuates tau pathology, and restores neuronal beta-catenin pathway function in an Alzheimer's disease model. J Immunol 187, 6539-6549.

[48] Weaver JD, Huang MH, Albert M, Harris T, Rowe JW, Seeman TE (2002) Interleukin-6 and risk of cognitive decline: MacArthur studies of successful aging. Neurology 59, 371-378.

[49] Lin YP, Thibodeaux CH, Pena JA, Ferry GD, Versalovic J (2008) Probiotic Lactobacillus reuteri suppress proinflammatory cytokines via c-Jun. Inflamm Bowel Dis 14, 1068-1083.

[50] Mencarelli A, Distrutti E, Renga B, D'Amore C, Cipriani S, Palladino G, Donini A, Ricci P, Fiorucci S (2011) Probiotics modulate intestinal expression of nuclear receptor and provide counter-regulatory signals to inflammation-driven adipose tissue activation. PLoS One 6, e22978.

[51] Kaur H, Nagamoto-Combs K, Golovko S, Golovko MY, Klug MG, Combs CK (2020) Probiotics ameliorate intestinal pathophysiology in a mouse model of Alzheimer's disease. Neurobiol Aging 92, 114-134.

[52] Fedorak RN, Feagan BG, Hotte N, Leddin D, Dieleman LA, Petrunia DM, Enns R, Bitton A, Chiba N, Pare P, Rostom A, Marshall J, Depew W, Bernstein CN, Panaccione R, Aumais G, Steinhart AH, Cockeram A, Bailey RJ, Gionchetti P, Wong C, Madsen K (2015) The probiotic VSL\#3 has anti-inflammatory effects and could reduce endoscopic recurrence after surgery for Crohn's disease. Clin Gastroenterol Hepatol 13, 928-935 e922.

[53] Dai C, Zheng CQ, Meng FJ, Zhou Z, Sang LX, Jiang M (2013) VSL\#3 probiotics exerts the anti-inflammatory activity via PI3k/Akt and NF-kappaB pathway in rat model of DSS-induced colitis. Mol Cell Biochem 374, 1-11.

[54] Schachter J, Martel J, Lin CS, Chang CJ, Wu TR, Lu CC, Ko YF, Lai HC, Ojcius DM, Young JD (2018) Effects of obesity on depression: A role for inflammation and the gut microbiota. Brain Behav Immun 69, 1-8.

[55] Tillisch K, Labus J, Kilpatrick L, Jiang Z, Stains J, Ebrat B, Guyonnet D, Legrain-Raspaud S, Trotin B, Naliboff B, Mayer EA (2013) Consumption of fermented milk product with probiotic modulates brain activity. Gastroenterology 144, 1394-1401, 1401 e1391-1394.

[56] Ait-Belgnaoui A, Colom A, Braniste V, Ramalho L, Marrot A, Cartier C, Houdeau E, Theodorou V, Tompkins T (2014) Probiotic gut effect prevents the chronic psychological stress-induced brain activity abnormality in mice. Neurogastroenterol Motil 26, 510-520.

[57] Mohle L, Mattei D, Heimesaat MM, Bereswill S, Fischer A, Alutis M, French T, Hambardzumyan D, Matzinger P, Dunay IR, Wolf SA (2016) Ly6C(hi) monocytes provide a link between antibiotic-induced changes in gut microbiota and adult hippocampal neurogenesis. Cell Rep 15, 19451956. 